\title{
MODELO DE CENTRALIZAÇÃO DE ESTOQUES PARA A LOGÍSTICA DE SUPRIMENTO DA EXPLORAÇÃO E PRODUÇÃO DA PETROBRAS
}

\author{
Edmar Diniz de Figueiredo \\ UN-SEAL \\ Petrobras \\ Aracaju - SE \\ Maria Aparecida Cavalcanti Netto \\ MBLog ${ }^{\circledR}$ PROJECT / COPPE \\ Universidade Federal do Rio de Janeiro \\ Rio de Janeiro - RJ \\ Recebido em 01/2001, aceito em 09/2001 após 1 revisão
}

\begin{abstract}
Resumo
Neste trabalho são analisados alguns aspectos básicos da logística de suprimento do segmento upstream - Exploração e Produção (E\&P) de petróleo, da Petrobras. Nele é proposto um modelo para apoio à decisão de centralização de seus estoques de partes comuns. Foi pressuposto que seria possível reduzir os níveis de estoque por meio da consolidação de locais de estocagem do projeto de redes orientadas para a eficiência. A revisão da literatura permitiu selecionar o modelo apropriado para a situação observada na empresa, base para uma metodologia de gestão do seu suprimento de materiais e equipamentos. Foi feita sua aplicação em um estudo de caso do E\&P-Nordeste, cujos resultados indicam ser possível $30,6 \%$ de redução média no nível dos estoques de segurança, os custos da logística variando em função da política de estoques adotada, das distâncias entre os locais, das modalidades de transportes e da freqüência de consumo.
\end{abstract}

Palavras-chave: logística do petróleo, modelo de estoque, logística de suprimento, centralização.

\begin{abstract}
This paper analyses some key aspects of the supply logistics for the upstream segment (E\&P Exploration \& Production) of Brazilian oil giant Petrobras. It suggests a model providing support for the decision to centralize its common inventories, based on the assumption that these inventory levels could be lowered through consolidating the storage centers for the efficiency-driven networks project. A review of the literature led to the selection of the most appropriate model for the company's situation, forming the basis of a management methodology for its supplies of materials and equipment. This was applied to a case study at the E\&P-Northeast, whose results indicate the possibility of an average reduction of $30.6 \%$ in safety stocks levels; the logistics costs vary by stock policy adopted, distances between facilities, shipping options and consumption rates.
\end{abstract}

Keywords: oil logistics, inventory model, supply logistics, centralization. 


\section{Introdução}

Este trabalho tem o objetivo de apresentar um modelo de apoio à decisão de centralização de estoques que possa ser base de uma metodologia de gestão integrada do suprimento das unidades operacionais do segmento de Exploração e Produção de petróleo (E\&P) da gigante do setor petrolífero Petrobras. A partir da análise de dados e informações relativas aos itens estocados nas unidades enfocadas e a revisão de estudos similares sobre centralização e gerenciamento de estoques, selecionou-se um modelo adequado à logística de suprimento do E\&P-Nordeste. Dessa forma, esse vai orientar a escolha da localização de centrais de administração compartilhada de estoques com base principalmente nos custos logísticos por unidade da empresa. É pressuposto que seriam possíveis reduções de estoques de itens comuns pela consolidação da rede de instalações.

Dessa forma, inicialmente são apresentados resultados de uma revisão de modelos de centralização de estoques, realizada em journals e revistas de interesse em logística e na mídia eletrônica. Embora tenha sido feita tendo por base o período de 1992 a 1998, a busca referenciada evidenciou a relevância de publicações de períodos anteriores. $\mathrm{Na}$ seção seguinte é descrito o problema específico de gerência de suprimento do segmento upstream da empresa e o modelo selecionado para apoio à decisão de centralização. Em seguida, é apresentada a metodologia do estudo de caso realizado para definição de uma rede consolidada de instalações e os resultados nele observados. Finalmente, a última seção traz as principais conclusões do trabalho.

\section{Revisão da Literatura de Modelos de Centralização e Gerenciamento de Estoques}

Segundo Meredith (1992), existem diversas razões para se manter estoques, mas em geral eles podem ser agrupados em cinco funções básicas ou seja, (a) segurança; (b) antecipação para épocas e ou situações especiais; (c) fragmentação entre os vários estágios de produção; (d) em trânsito, e (e) cíclicos, necessários para permitir economias de escala. Estoques de segurança são mantidos para estabelecer uma margem de segurança ao longo de cadeias de abastecimento e de forma a minimizar as incertezas, associadas ao suprimento e/ou demanda, com o objetivo de garantir o funcionamento ininterrupto do processo produtivo. $\mathrm{O}$ estabelecimento dessa margem busca reduzir o risco que a empresa está disposta a assumir por causa da ocorrência de falta ou falha de estoque. $\mathrm{O}$ estoque de segurança em cada ponto de estocagem na cadeia de abastecimento depende da variabilidade da demanda da região que ele atende, da incerteza existente em relação ao lead time e do nível de serviço desejado pela empresa.

Segundo Zin \& Marmorstein (1990), os dois métodos mais utilizados para o cálculo do estoque de segurança são baseados no Sistema de Demanda (DS) e no Sistema de Previsão (FS). A diferença entre os dois sistemas é substancial. Resultados de simulações indicam que o FS, embora menos utilizado na literatura da área de logística, resulta em aproximadamente $15 \%$ menos estoque de segurança para assegurar o mesmo nível de serviço ao cliente que o DS. No método DS - Sistema de Demanda, o nível de estoque de segurança depende da variabilidade da demanda. Já no FS - Sistema de Previsão, esse nível depende da variabilidade dos erros de previsão da demanda. A formulação para o cálculo do estoque de segurança no sistema FS é similar ao DS. Entretanto, no DS a previsão implícita para cada período é a demanda média. Como resultado, o desvio padrão da demanda equivale ao desvio padrão da "previsão" de erros. O FS é mais complexo e caro de implementar que o DS, mas potencialmente mais eficiente, pois requer a estimação do erro de previsão, o que é 
obtido usando aproximações baseadas por exemplo na teoria de regressão ou análise de séries temporais, para prever a demanda para cada período futuro.

Zin et al. (1989) utilizam a abordagem do sistema DS para o cálculo do estoque de segurança por meio da expressão $\mathrm{SS}=\mathrm{k} \sigma$, onde: $\mathrm{k}=$ fator de segurança associado ao nível de serviço desejado; $\sigma=$ desvio padrão da demanda; e $\mathrm{SS}=$ estoque de segurança. A grande vantagem de expressá-lo dessa forma deriva do fato de existirem tabelas que apresentam a probabilidade de faltar estoque em função do fator de segurança $\mathrm{k}$, medido em números de desvios padrão em relação à média da amostra de dados. Lambert \& Stock (1993) apresentam uma tabela relacionando os diversos valores para $\mathrm{k}$ aos valores assumidos por suas funções de densidade e probabilidade e suas expectâncias parciais (Figueiredo, 1999). Essa tabela foi originalmente desenvolvida por Brown (1967) e os valores nela contidos foram obtidos a partir da premissa de que a demanda e os erros de sua previsão apresentam uma distribuição normal. Embora tal premissa pareça atender à maioria dos casos práticos, é ressalvado que, quando tal não ocorrer, os valores tabelados poderiam ser recalculados para a distribuição acertada, sem prejuízo para a especificação funcional do cálculo do estoque de segurança, válida para qualquer distribuição da demanda e dos erros de sua estimativa.

A revisão da literatura indica como as primeiras referências sobre centralização de estoques os livros de Starr \& Miller (1962) e Brown (1967). Só anos mais tarde aplicações dos modelos de centralização baseadas na famosa "lei da raiz quadrada" apareceram nos livrostexto da área de logística como, por exemplo, Heskett et al. (1973) e Smykay (1973). Heskett et al. (1973) enumeram, entre outros pontos, os maiores benefícios das estratégias de centralização versus estratégias de descentralização e os fatores responsáveis por cada um desses. Smykay (1973) observa que o estoque de segurança armazenado em um único ponto centralizado é igual à razão do estoque total de segurança dos vários locais possíveis pela raiz quadrada do número total de localizações. Embora a comprovação prática da "lei da raiz quadrada" indique que o total de estoque em um sistema é proporcional à raiz quadrada do número de localizações onde o produto pode ser estocado, em nenhuma das referências acima é feita, explicitamente, sua demonstração formal.

Maister (1976) formaliza a "lei da raiz quadrada" e estabelece que, em geral, a relação entre estoque centralizado e descentralizado é igual a: $\frac{\sqrt{m}}{\sqrt{n}}$, sendo $\mathrm{m}=$ número de localizações após a consolidação e $\mathrm{n}=$ número de localizações anteriores à consolidação. Segundo o autor, essa lei pode ser aplicável tanto ao cálculo da redução do estoque de segurança, quanto ao cálculo de estoques de manutenção quando for empregado o lote econômico para pedido de ressuprimentos (EOQ). Ele complementa que, quando o estoque de manutenção está diretamente relacionado com a demanda média, a redução obtida com a aplicação da "lei da raiz quadrada" não é pertinente. Para a dedução da lei, Maister (1976) estabelece as seguintes hipóteses: as demandas de cada local não são correlacionadas e a variabilidade da demanda é a mesma para todas as localizações. Uma aproximação da programação dinâmica para alocar estoques de manutenção e de segurança a várias localizações, comparando o custo total de estoque centralizado versus descentralizado, segundo hipóteses menos restritivas que as de Maister (1976) é feita em 1978 por Das (Das \& Tyagi, 1997).

Eppen (1979) estuda os efeitos de centralização nos custos de manutenção e nos custos de penalidades por transferências entre localizações no problema conhecido na literatura como do "jornaleiro de múltiplas localizações". O autor deduz em seu trabalho uma expressão para o custo total esperado ao consolidar demandas de várias instalações. Segundo ele, o custo 
total esperado é uma função dos parâmetros da demanda de cada localização como média, variância e coeficientes de correlação. A modelagem de Eppen (1979) permite mostrar que o custo esperado de manutenção de estoques e das penalidades, em sistemas descentralizados, é maior do que em sistemas centralizados. Essa modelagem permite observar também que se as demandas são idênticas e não correlacionadas, esses custos aumentam com a raiz quadrada do número de locais de armazenagem ou seja, com o "tamanho da armazenagem" considerada. Outros autores como Chen \& Lin (1989) e Chang \& Lin (1991) utilizam a formulação do problema do "jornaleiro de múltiplas localizações” em suas modelagens.

Ballou (1981) faz uma avaliação empírica da "lei da raiz quadrada" para o estoque total de segurança, por meio da análise de regressão, usando dados de várias indústrias. Os parâmetros da equação de regressão mostram que o nível de agregação do estoque difere de indústria para indústria. $\mathrm{O}$ autor apresenta resultados de diferentes empresas em setores industriais variados, demonstrando que a "lei da raiz quadrada" é normalmente muito otimista em relação às reduções do estoque.

Zin et al. (1989) desenvolvem um modelo abrangente, do qual o da "lei de raiz quadrada" é caso particular. Aquele foi denominado Portfolio Effect por medir o percentual possível de redução no estoque total de segurança por meio da consolidação de múltiplas localizações em um número reduzido delas. O Portfolio Effect é medido como uma função de duas variáveis: a correlação dos fluxos demandados da série histórica de consumo entre dois locais (coeficiente de correlação de Pearson) e a magnitude, definida como a relação entre os desvios padrão do consumo entre duas localizações. Os autores definem o modelo de Portfolio Effect para os casos de consolidar dois e três locais de estocagem e sugerem que os valores resultantes da modelagem podem ser usados para identificar oportunidades de redução no estoque de segurança em um ambiente de múltiplas localizações.

A medida do Portfolio Effect das consolidações está basicamente relacionada à noção de agregação de estoques. Em uma rede de múltiplas localizações, desequilíbrios na disponibilidade dos estoques por aleatoriedade da demanda nas diferentes localizações podem ser compensados por meio de transferências entre locais adjacentes. A agregação de localizações é também usada em ambientes não industriais, em situações como a transferência de pacientes entre hospitais. O princípio básico que explica o Portfolio Effect está baseado na noção de agregação de riscos que é utilizado na literatura sobre análise de partes comuns. Essa trata da medição do efeito do grau de padronização de itens componentes no estoque total. Essa literatura avalia o uso de um único item de reposição idêntico como um sobressalente em substituição a um conjunto de vários itens componentes de forma a se conseguir redução nos estoques de segurança.

Evers (1995) amplia a análise da "lei da raiz quadrada". Para isso analisa o problema dos estoques mantidos utilizando o lote econômico ou determinando a quantidade a ser pedida a partir da demanda média prevista para o período do lead time. O modelo de Evers (1995) é deduzido a partir de hipóteses que são pouco prováveis de serem observadas na prática, limitando a sua operacionalidade. Em sua análise, o autor conclui que seriam necessárias novas pesquisas para avaliar o realismo das hipóteses estabelecidas e se a "lei da raiz quadrada" geraria um bom estimador quando pelo menos algumas ou todas as hipóteses estivessem presentes, semelhantes àquela de Ballou (1981).

Tallon (1993) estende o modelo de Zin et al. (1989) estudando o impacto da centralização dos estoques em uma rede consolidada quando a demanda e o lead time são incertos. $\mathrm{O}$ autor propõe um modelo para calcular a redução no estoque total de segurança, considerando 
vários padrões alternativos da demanda e do lead time. Em suas conclusões avalia que a centralização reduz o nível dos estoques de segurança num ambiente de incerteza. Porém, um maior grau de centralização pode aumentar as distâncias para os mercados e, conseqüentemente, prejudicar o atendimento ao cliente. Segundo o autor, essas reduções no nível de serviço poderiam ser minimizadas por meio de melhorias no sistema de transportes ou no desempenho do processamento de pedidos. As incertezas enfrentadas pela empresa poderiam ser reduzidas por meio de acordos contratuais com clientes e fornecedores. A redução de incertezas pode fazer com que o grau de centralização seja maior, possibilitando uma maximização nos resultados obtidos pela consolidação dos estoques.

Mahmoud (1992) apresenta um estudo estendido ao realizado por Zin et al. (1989). Na verdade, o modelo de Mahmoud (1992) complementa o de Zin et al. (1989), ao permitir que se calcule a quantidade reduzida no estoque de segurança e se selecione o(s) melhor(es) local(is) para a centralização dos estoques com base nos custos logísticos, por meio de um modelo de otimização combinatória. O autor modela o caso geral do "problema de seleção do esquema ótimo de consolidação" com uma estrutura funcional semelhante àquela dos modelos de particionamento e p-medianas. No caso de limites, como exigências de consolidação em grupos de no máximo duas instalações, Mahmoud (1992) conclui que o problema é do tipo network weighted matching.

Finalmente, Das \& Tyagi (1997) apresentam um modelo para análise formal da decisão de centralização de estoques em uma perspectiva mais ampla. Os autores elaboram vários cenários relativos aos componentes do custo logístico e analisam os efeitos individuais ou combinados desses no grau de centralização do sistema estudado, composto por três instalações e oito clientes, usando um modelo de otimização. Seu objetivo é determinar o grau ótimo de centralização por meio de uma análise das inter-relações dos custos de estocagem e transportes.

\section{O Estudo de Caso}

\subsection{A Situação em Estudo}

A atividade de Exploração e Produção da indústria de petróleo, denominada segmento upstream, abrange as áreas de prospecção de jazidas, desenvolvimento de reservas, produção de petróleo e gás natural. Pertencendo o E\&P a uma atividade de mineração - pesquisa e lavra de hidrocarbonetos, a localização de suas instalações produtivas depende de onde sejam descobertas as jazidas. Cada campo, ou grupo de campos de petróleo, se constitui em uma unidade de extração, cuja vida útil e volume produzido dependem das características do reservatório até que ele seja depletado. As atividades de pesquisa e desenvolvimento de reservas caracterizam-se pelo uso intensivo de capital e elevado risco de insucesso econômico. Essas características refletem-se na busca da maximização da eficiência operacional na pesquisa exploratória, na perfuração de poços e também na fase de produção, por meio da operação contínua e da máxima utilização da capacidade instalada. Segundo Hansen (1996), "essas características tornam essa indústria objeto da filosofia de economia de escala isto é, busca de elevados fatores de utilização e de minimização das interrupções de produção com vistas à redução dos custos unitários dos produtos". Uma conseqüência dessa filosofia de operação é a geração de uma grande demanda por atividades de suporte, como serviços logísticos em geral e de manutenção, que na indústria do petróleo influenciam fortemente os custos totais de produção. 
Em virtude do grande impacto dos custos logísticos nas atividades do E\&P e da perspectiva de ganhos com melhorias na gestão dos processos a elas relacionados, a atividade de gerência de material ganhou destaque na Petrobras. O custo médio agregado pela logística de suprimento ao segmento E\&P é de US\$ 0,44 por barril produzido, em valores de setembro de 1998. Embora o custo médio de produção do E\&P seja de US\$ 5,75 por BOE (Barril de Óleo Equivalente), há grande variação entre unidades. Por exemplo, os maiores custos são relativos às áreas de produção do Recôncavo (Bahia), Sergipe-Alagoas (SEAL), Espírito Santo (ES) e Rio Grande do Norte e Ceará (RNCE) ou seja US\$11,62; US\$ 10,03; US\$ 8,80; e US\$ 6,54, respectivamente, por BOE. Os menores são relativos às unidades UN-SUL e UN-BC (Bacia de Campos). O posicionamento dessas áreas pode ser visualizado pelo mapa da Figura 1. Da mesma forma, há variação do valor agregado ao barril de petróleo pela logística de suprimento por unidade operacional, alcançando valores por barril produzido de US\$ 1,01 na unidade do Espírito Santo e de US\$ 0,90; US\$ 0,84 e US\$ 0,61 nas três unidades do Nordeste, UN-SEAL, BA e RNCE, respectivamente.

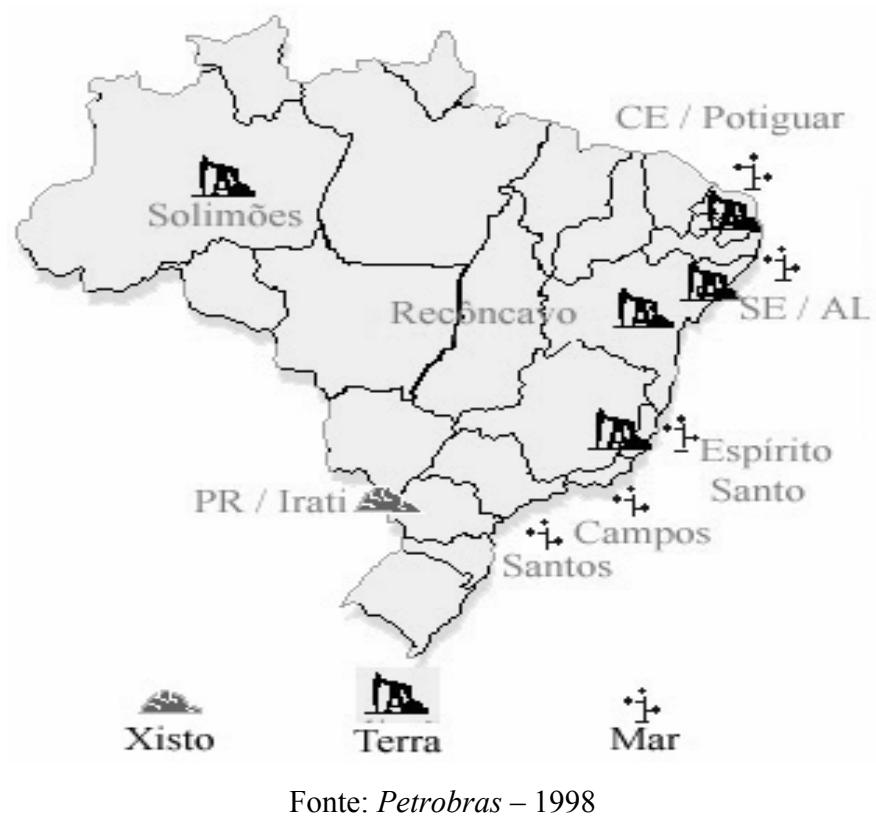

Figura I - Áreas de Produção de Petróleo e Gás da Petrobras no Brasil

Dentre os itens que compõem o custo total de suprimento de materiais, a manutenção de estoques tem o maior potencial ou seja, $67 \%$ do total, isso avaliando-se apenas o segundo trimestre de 1998, conforme relatório do Sermat (1998). A participação de demais componentes de custo é a seguinte: a) pessoal: $16 \%$, b) serviço de terceiros: $6 \%$, c) instalações: 3\%, d) material de consumo: 1\%, e) overhead: $7 \%$, e f) encargos: $1 \%$.

$\mathrm{O}$ atual sistema de gestão de estoques do E\&P/Petrobras é descentralizado com determinados itens podendo ser estocados em várias unidades, com parâmetros de ressuprimento predefinidos e estoques de segurança mantidos em cada uma delas. As compras também são feitas de forma descentralizada, sendo que para alguns itens o fornecedor é único. Essa 
situação duplica atividades realizadas dentro do mesmo segmento e diminui uma das forças competitivas da empresa, o seu poder de compra, isso considerando somente o E\&P.

Buscando economias de escala, o planejamento de estratégias logísticas estimula o projeto de redes orientadas para a eficiência baseadas na consolidação de locais de estocagem. Visando desenvolver um instrumento de suporte gerencial para a revisão e o estabelecimento de políticas de gerência de estoques para o segmento do E\&P, foi realizado um estudo de caso para o gerenciamento dos estoques das suas unidades operacionais do Nordeste. A abordagem adotada nesse estudo de caso está alinhada às diretrizes de planejamento da alta administração da empresa que vem procurando estimular o fortalecimento das parcerias internas e o melhor aproveitamento do potencial de cada unidade, ao mesmo tempo procurando reduzir custos e fazer prevalecer o poder de compra.

A área considerada no estudo de caso restringiu-se à região Nordeste por motivo de facilidade de obtenção de dados e conhecimento prévio da situação vigente nas unidades operacionais do E\&P nessa região. Além disso, as três unidades UN-BA, UN-SEAL e UN-RNCE, responsáveis pela Exploração e Produção respectivamente na Bahia, Sergipe e Alagoas, e Rio Grande do Norte e Ceará, apresentam características muito similares de produção em termos de localização dos poços, com a grande maioria deles localizados em terra e com equipamentos idênticos para extração do petróleo. A área estudada contém duas das unidades operacionais com os maiores custos de produção entre aquelas que compõem o E\&P, justificando o planejamento de ações de melhoria que visem redução nos custos de produção. A produção média das três unidades da região Nordeste é de 245.084 barris por dia, o que equivale a $21 \%$ da produção nacional (Figueiredo, 1999). Os estoques dessas unidades totalizam o valor de US\$219.896.000,00, com 60.197 itens estocados.

\subsection{O Modelo Selecionado para Apoiar as Decisões de Centralização de Estoques}

Entre os modelos de centralização de estoques da literatura foi selecionado o modelo de Zin et al. (1989) estendido por Mahmoud (1992) para ser aplicado ao estudo do caso realizado. O modelo desenvolvido por Maister (1976) seria também aplicável ao problema em estudo, mas ele é na verdade um caso particular do modelo de Zin et al. (1989). A seleção desse modelo foi reforçada pelos seguintes pontos:

1. ele é mais abrangente pois permite selecionar as melhores localizações para consolidação de estoques;

2. ele analisa o problema de forma integrada, considerando os vários componentes do custo total, como os custos de transportes, de manutenção de estoques e de processamento de pedidos e, além disso;

3. ele não exige grandes recursos computacionais para a solução do problema.

São apresentadas a seguir a generalização do modelo de Zin et al. (1989) e as extensões do modelo de Mahmoud (1992).

\section{- Generalização do Modelo de Zin et al. (1989)}

Zin et al. (1989) definem o efeito da consolidação de instalações existentes em subconjuntos I' por meio do modelo Portfolio Effect. As seguintes notações são usadas para generalização desse modelo nas equações (1) a (18): 
$\mathrm{I}=$ Conjunto de $\mathrm{n}$ locais de estoque, indexados por $\mathrm{i}$;

I' = Subconjuntos de locais de estoque a serem consolidadas, I' $\subset \mathrm{I},\left|\mathrm{I}^{\prime}\right|=\mathrm{m}$, $2 \leq \mathrm{m} \leq \mathrm{n}, \mathrm{I} \prime \neq \varnothing ;$

$\mathrm{SS}_{\mathrm{i}}=$ Estoque de segurança armazenado no local $\mathrm{i}, \mathrm{i} \in \mathrm{I}$;

$\mathrm{SS}_{\mathrm{I}},=\mathrm{O}$ estoque de segurança central para os locais de estoques consolidados I';

$\mathrm{PE}_{\mathrm{I}},=\mathrm{O}$ Portfolio Effect da consolidação dos locais de estoque que compõem I';

$\mathrm{k}=$ Fator do estoque de segurança, determinado gerencialmente, assumido como idêntico em todos os locais ;

$\sigma_{\mathrm{i}}=$ Desvio padrão da demanda no local $\mathrm{i}, \mathrm{i} \in \mathrm{I}$;

$\sigma_{\mathrm{I}}{ }^{\prime}=\mathrm{O}$ desvio padrão central da demanda consolidada dos locais que compõem I';

$\mathrm{M}_{\mathrm{ij}}=\mathrm{A}$ magnitude relativa entre o desvio padrão da demanda nos locais $\mathrm{i}$ e $\mathrm{j}$, $\mathrm{M}_{\mathrm{ij}}=\frac{\sigma_{i}}{\sigma_{j}}$ onde $\sigma_{\mathrm{i}} \geq \sigma_{\mathrm{j}}$ e $\sigma_{\mathrm{j}} \neq 0$;

$\rho_{\mathrm{ij}}=$ O coeficiente de correlação de Pearson das demandas entre os locais i e j, i, j $\in \mathrm{I}$.

A equação (1) define o Portfolio Effect $\left(\mathrm{PE}_{\mathrm{I}}\right)$ por meio da divisão da taxa de redução no volume total do estoque de segurança após a consolidação por esse mesmo volume na situação anterior a ela.

$$
\mathrm{PE}_{\mathrm{I}^{\prime}}=\frac{\sum_{i \in I^{\prime}} S S_{i}-S S_{I^{\prime}}}{\sum_{i \in I^{\prime}} S S_{i}}
$$

Para facilitar a generalização do modelo, reescreve-se a equação (1) como:

$$
\mathrm{PE}_{\mathrm{I}^{\prime}}=1-\frac{\mathrm{SS}_{\mathrm{I}^{\prime}}}{\sum_{\mathrm{i} \in I^{\prime}} \mathrm{SS}_{\mathrm{i}}}
$$

O estoque de segurança em um local i qualquer é calculado como:

$$
\mathrm{SS}_{\mathrm{i}}=\mathrm{k} \sigma_{\mathrm{i}}
$$

Assumindo que $\mathrm{k}$ é o mesmo para cada local de estoque $\mathrm{i} \in \mathrm{I}$ e para todas consolidações possíveis I' $\in \mathrm{I}$, a equação (2) pode ser reescrita como:

$$
\mathrm{PE}_{\mathrm{I}}=1-\frac{\sigma_{\mathrm{I}^{\prime}}}{\sum_{\mathrm{i} \in \mathrm{I}^{\prime}} \sigma_{\mathrm{i}}}
$$

O desvio padrão da demanda no local onde é consolidado o estoque é definido estatisticamente (Mood et al., 1974) como:

$$
\sigma_{\mathrm{I}}=\sqrt{\sum_{i \in I^{\prime}} \sigma_{i}^{2}+2 \sum_{\substack{i \\ i, j \in I^{\prime} \\ i<j}} \sum_{j} \sigma_{i} \sigma_{j} \rho_{i j}}
$$

Substituindo a equação (5) na equação (4), 


$$
\mathrm{PE}_{\mathrm{I}^{\prime}}=1-\frac{\sqrt{\sum_{i \in I^{\prime}} \sigma_{i}^{2}+2 \sum_{\substack{i \\ i, j \\ i<j}} \sum_{j} \sigma_{i} \sigma_{j} \rho_{i j}}}{\sum_{\mathrm{i} \in \mathrm{I}^{\prime}} \sigma_{\mathrm{i}}}
$$

Usando a definição de magnitude $\mathrm{M}_{\mathrm{ij}}$, a equação (6) pode ser reescrita como:

$$
\mathrm{PE}_{\mathrm{I}^{\prime}}=1-\frac{\sqrt{\sum_{i \in I^{\prime}} M_{i m}^{2}+2 \sum_{\substack{i \\ i, j \in I^{\prime} \\ i<j}} \sum_{i m} M_{i m} M_{j m} \rho_{i j}}}{\sum_{i \in I^{\prime}} M_{i m}}
$$

Na equação (7) os elementos de I' são indexados tal que $\sigma_{\mathrm{m}}=\max \left[\sigma_{\mathrm{i}} ; \forall \mathrm{i} \in \mathrm{I}^{\prime}\right]$.

A equação (7) calcula o ganho possível, de forma genérica, com a centralização de estoques numa rede com múltiplas localizações. Por exemplo, no problema da análise da consolidação de $\mathrm{n}=3$ unidades são possíveis as seguintes combinações: 1-2, 1-3, 2-3 e 1-2-3. Para cada uma dessas consolidações, os valores percentuais de redução no estoque total de segurança são calculados conforme as expressões (8) a (11):

$$
\begin{aligned}
& \mathrm{PE}_{12}=1-\frac{\sqrt{M_{12}^{2}+1+2 M_{12} \rho_{12}}}{M_{12}+1} \\
& \mathrm{PE}_{13}=1-\frac{\sqrt{M_{13}^{2}+1+2 M_{13} \rho_{13}}}{M_{13}+1} \\
& \mathrm{PE}_{23}=1-\frac{\sqrt{M_{23}^{2}+1+2 M_{23} \rho_{23}}}{M_{23}+1} \\
& \mathrm{PE}_{123}=1-\frac{\sqrt{M_{13}^{2}+M_{23}^{2}+1+2 M_{13} M_{23} \rho_{12}+2 M_{13} \rho_{13}+2 M_{23} \rho_{23}}}{M_{13}+M_{23}+1}
\end{aligned}
$$

\section{- Cálculo da Quantidade Reduzida no Estoque de Segurança pelo Modelo Portfolio Quantity Effect}

O modelo Portfolio Effect desenvolvido por Zin et al. (1989) permite obter uma medida do percentual de redução no estoque total de segurança conseguido por meio da consolidação de estoques de múltiplas localizações em um único ponto. Como os níveis de estoques de segurança armazenados em cada local $\left(\mathrm{SS}_{\mathrm{i}}\right)$ diferem uns dos outros, o ganho conseguido com a consolidação deve, de fato, ser medido em termos de redução nas quantidades. Mahmoud (1992) estendeu esse modelo chamando-o de Portfolio Quantity Effect (PQE), calculando a quantidade total da redução na quantidade do estoque de segurança, conseguida com a sua centralização, pela equação (12).

$$
\mathrm{PQE}_{\mathrm{I}^{\prime}}=\mathrm{PE}_{\mathrm{I}^{\prime}}\left(\sum_{i \in I^{\prime}} S S_{i}\right)
$$




\section{- Cálculo do Ganho na Centralização de Estoque pelo Modelo Portfolio Cost Effect}

As decisões tomadas a partir de modelos de centralização de estoques em geral dizem respeito à quantidade total reduzida no estoque de segurança. Na prática, o gerente do sistema logístico está interessado em estudar também o efeito dessas decisões no custo total do sistema e no nível de serviço ao cliente. A hipótese adotada no modelo estendido por Mahmoud (1992) é de que a instalação centralizadora de estoques será alocada a uma das localizações já existentes.

Uma análise mais abrangente do problema deveria incluir a determinação de local(is) ótimo(s) onde se localizaria(m) a(s) instalação(ões) centralizadora(s) com base em alguns fatores tais como: a distribuição espacial da demanda, a média e a variância do tempo em trânsito e o nível de serviço ao cliente. Os modelos clássicos da teoria de localização podem ser usados na determinação dessa(s) instalação(ões) centralizadora(s). Mirchandani \& Francis (1990) apresentam um trabalho amplo sobre a teoria de localização de instalações.

Para selecionar o local da centralização são calculados os componentes do custo total associado a essa decisão com base no modelo denominado por Mahmoud (1992) de Portfolio Cost Effect (PCE). Assim, para cada subconjunto I' de locais consolidados calcula-se o Portfolio Cost Effect ( $P C E_{I^{\prime}}^{r}$ ) para localizar a instalação centralizadora $\mathrm{em} \mathrm{r}, \mathrm{r} \in \mathrm{I}$ '. No caso geral, em que $2<\mathrm{m} \leq \mathrm{n}$, o número total de operações para avaliações do $P C E_{I^{\prime}}^{r}$ é igual a $\sum_{m=2}^{n} m\left(\begin{array}{l}n \\ m\end{array}\right)$, onde o termo $\left(\begin{array}{l}n \\ m\end{array}\right)$ é o coeficiente binomial.

O modelo PCE mede a redução no custo total do sistema logístico, o qual inclui os seguintes componentes:

- Custo de Manutenção de Estoque ( $\left.\mathrm{PHCE}_{\mathrm{I}^{\mathrm{r}}}\right)$;

- Custo de Transportes $\left(\mathrm{PTCE}_{\mathrm{I}}{ }^{\mathrm{r}}\right)$;

- Custo de Investimento em Instalações $\left(\mathrm{PICE}_{\mathrm{r}}{ }^{\mathrm{r}}\right.$ );

- Custo de Processamento de Pedido ( $\mathrm{PPCE}_{\mathrm{I}}{ }^{\mathrm{r}}$ ).

O ganho com a consolidação, medido através do modelo Portfolio Cost Effect (PCE), pode ser calculado conforme a expressão (13):

$$
\operatorname{PCE}_{\mathrm{I}}{ }^{\mathrm{r}}=\mathrm{PHCE}_{\mathrm{I}}{ }^{\mathrm{r}}+\mathrm{PTCE}_{\mathrm{I}}{ }^{\mathrm{r}}+\mathrm{PICE}_{\mathrm{I}}{ }^{\mathrm{r}}+\mathrm{PPCE}_{\mathrm{I}}{ }^{\mathrm{r}}
$$

No cálculo do valor máximo do Portfolio Cost Effect obtido por $\mathrm{PCE}_{\mathrm{I}}{ }^{*}=\operatorname{Max}\left[P C E_{I^{\prime}}^{r}\right], \mathrm{o}$ número total de avaliações por subconjunto I' $\left(\mathrm{PCE}_{\mathrm{I}^{\mathrm{r}}}{ }^{\mathrm{r}}\right)$ pode ser reduzido para $\sum_{m=2}^{n}\left(\begin{array}{l}n \\ m\end{array}\right)$, se forem tomados apenas os valores correspondentes às instalações centralizadoras por subconjunto ou seja, aquelas que fornecem os maiores $\mathrm{PCE}_{\mathrm{I}}{ }^{\mathrm{r}}$.

\section{- Seleção do Esquema Ótimo de Consolidação (OCS)}

$\mathrm{O}$ maior ganho total nos valores de $\mathrm{PCE}_{\mathrm{I}}{ }^{*}$ é obtido com o esquema ótimo de consolidação (S*). Mahmoud (1992) definiu o problema de selecionar esse esquema da seguinte forma: 
$<$ OCS $>\quad$ Maximizar $\sum_{I_{k}^{\prime} \in C} P C E_{I^{\prime} K_{K}}^{*}$

$$
\text { Sujeito a: } \begin{aligned}
\bigcup_{I^{\prime}} \in S & I^{\prime}{ }_{k}=I ; \\
& \mathrm{I}_{\mathrm{i}} \cap \mathrm{I}^{\prime}{ }_{\mathrm{j}}=\varnothing, \\
& \forall \mathrm{I}^{\prime}{ }_{\mathrm{i},} \mathrm{I}^{\prime}{ }_{\mathrm{j}} \in \mathrm{S} .
\end{aligned}
$$

onde:

$\mathrm{S}$ é o conjunto de esquemas de consolidação, composto das consolidações I' ${ }_{1}, \mathrm{I}_{2},{ }_{2}, \ldots, \mathrm{I}_{\mathrm{Z}}$, que satisfazem às duas condições abaixo:

$$
\begin{aligned}
& \text { (a) } \mathrm{I}^{\prime}{ }_{1} \cup \mathrm{I}^{\prime}{ }_{2} \cup, \ldots, \cup \mathrm{I}^{\prime}{ }_{\mathrm{Z}}=\mathrm{I} ; \\
& \text { (b) } \mathrm{I}^{\prime}{ }_{\mathrm{i}} \cap \mathrm{I}_{\mathrm{j}}{ }_{\mathrm{j}}=\varnothing \forall \mathrm{I}^{\prime}{ }_{\mathrm{i}}, \mathrm{I}_{\mathrm{j}} \in \mathrm{S} ; \mathrm{i} \neq \mathrm{j},
\end{aligned}
$$

sendo que pela condição (a) cada instalação de I pertence a uma consolidação e, pela condição (b), cada instalação de I pertence no máximo a um desses subconjuntos;

$\mathrm{C}$ é o subconjunto de todas as consolidações possíveis, sendo $\mathrm{C}=\left\{\mathrm{I}_{1}{ }_{1}, \mathrm{I}_{2}{ }_{2}, \ldots, \mathrm{I}_{\mathrm{k}}, \ldots, \mathrm{I}_{\mathrm{N}}\right\}$, e $\mathrm{N}$ o número de consolidações possíveis distintas indexadas por $\mathrm{k}$, de forma que $|\mathrm{C}|=\mathrm{N}$, e $\mathrm{N}=\sum_{m=2}^{n}\left(\begin{array}{l}n \\ m\end{array}\right)$.

A estrutura do problema definido pelas equações (14), (15) e (16) é similar ao problema de otimização combinatória conhecido na literatura como problema do particionamento.

\subsection{Metodologia do Estudo de Caso}

O problema de gerenciamento dos estoques nas unidades operacionais do Nordeste do E\&P/Petrobras refere-se a um problema de consolidação da rede de instalações para estocagem de itens de suporte ao processo produtivo ou seja: Exploração e Produção de petróleo. Sua solução constitui a base para a definição de uma política ótima para a logística de suprimento. As etapas para a análise do problema são apresentadas a seguir:

- Levantamento e Análise dos Dados;

- Modelagem do Problema de Consolidação de Estoques;

- Solução do Problema de Consolidação de Estoques;

- Análise dos Resultados do Estudo de Caso.

\section{- Levantamento e Análise dos Dados}

A etapa de levantamento de dados foi realizada no período de 01 de julho de 1998 a 30 de setembro de 1998. O objetivo inicial foi identificar os itens comuns às três unidades operacionais do E\&P-Nordeste, utilizando para isso o banco de dados do Sistema Único de Material (SUM), por unidade operacional da empresa, via endereços TCP/IP das suas redes internas de informática. 
O Manual de Suprimento de Material (MSM) da empresa classifica os itens em estoque por tipo de gestão, observando a sua situação: Estoque Ativo ou Inativo; Disponível ou Alienável; a sua aplicação: Consumo, Aplicação Específica, Especial, ou Análise e o tipo de demanda: Probabilística, Incerta, Programada, ou Eventual. Assim, os itens são classificados como: Consumo (C), Imediato (I), Especial (E), Fim (F), Obra (O), Manutenção (M), Análise (A), Disponível (D), Alienável (V) ou Eventual (L) (Figueiredo, 1999).

Os itens analisados, para os quais se visou reduzir o estoque pela consolidação de locais do projeto de redes orientadas para a eficiência, foram limitados àqueles classificados como do tipo Consumo (C) pela empresa ou seja: Estoque Ativo/Consumo e Demanda Probabilística. Esses itens são caracterizados no MSM como "enquadrados em modelos de controle aprovados, com parâmetros implantados ou calculados conforme política de estoques da unidade operacional, cujo ressuprimento é realizado sem consulta ao cliente, após análise do histórico de consumo e dos níveis de estoque". A Petrobras, para esses itens, utiliza o sistema de revisão contínua $(\mathrm{s}, \mathrm{Q})$ para o controle do estoque, sendo s o ponto de pedido e $\mathrm{Q}$ o tamanho do pedido de reposição (Silver \& Peterson, 1985).

Para os itens selecionados de demanda tipo Consumo (C), foi feita uma outra classificação, seguindo o critério de Pareto ou ABC (Lambert \& Stock, 1993) e limitou-se o estudo aos itens classe A, correspondendo a $20 \%$ dos itens selecionados. Figueiredo (1999) contém a listagem completa de todos os itens comuns às três unidades operacionais e o histórico de consumo dos itens classe A, referente ao período de 01 de janeiro de 1996 a 30 de setembro de 1998, com a descrição destes itens, seus preços unitários e os valores em estoque em 30 de setembro de 1998 de cada um deles.

A partir do histórico do consumo dos itens classe A por unidade, foi feita uma análise dos dados de base estatística, utilizando o software BESTFIT (Palisade). Constatou-se que, tanto para o consumo individual quanto para a soma dos consumos nas três unidades, a hipótese de que a distribuição de consumo por item segue uma distribuição normal poderia ser aceita. Como para alguns itens não houve consumo observado em todos os meses no período de tempo em referência, adotou-se como critério para preparar os dados para a análise estatística considerar apenas os meses em que foi registrado consumo. Quando em um mês o item não foi consumido em nenhuma das três unidades, esse mês foi descartado da série histórica de consumo. Esse critério é justificado pela avaliação do impacto do coeficiente de Pearson, que mede a correlação entre demandas no Portfolio Effect, pois caso o critério não fosse considerado, o percentual de redução no estoque de segurança seria aumentado.

\section{- Modelagem do Problema de Consolidação de Estoques}

A modelagem do problema de consolidação baseou-se no modelo de centralização selecionado na literatura pois esse define, em última instância:

- A quantidade total reduzida no estoque de segurança;

- O ganho anual conseguido com a consolidação pela redução do custo logístico;

- O melhor local para centralizar o estoque.

O ganho total resultante da redução no estoque de segurança pela consolidação dos estoques em um número reduzido de instalações centralizadoras pode ser calculado pelo modelo Portfolio Cost Effect $\left(\mathrm{PCE}_{\mathrm{I}}{ }^{\mathrm{r}}\right)$, composto pelos componentes de custo de manutenção de estoques, de transportes, de processamento de pedidos e de investimento. Esse último não 
será considerado, no caso em estudo, pelo fato de que o local da centralização será em uma das unidades preexistentes, que já possui toda a infra-estrutura implantada e armazéns atualmente com capacidade física e operacional ociosa, sem necessidade de novos investimentos em expansão.

Esses armazéns foram construídos num período em que as unidades estavam em franca expansão (década de 1970) e havia uma grande dificuldade para importação, obrigando a Petrobras a exigir dos fabricantes o fornecimento de sobressalentes para um horizonte de dois anos de operação, quando comprava um equipamento novo. Além disso, a infraestrutura de comunicação e transportes necessária às operações da empresa era muito deficiente, resultando em ciclos de pedidos muito longos.

O cálculo da quantidade reduzida no estoque de segurança e dos componentes do custo logístico do Portfolio Cost Effect são apresentados nos subitens a seguir.

\section{a) Cálculo da Quantidade Reduzida no Estoque de Segurança}

A quantidade total da redução do estoque de segurança por subconjunto de locais a serem consolidados I' é calculada pelo modelo Portfolio Quantity Effect $\left.\left(\mathrm{PQE}_{\mathrm{I}}\right)^{\prime}\right)$ representado por:

$$
\mathrm{PQE}_{\mathrm{I}^{\prime}}=\mathrm{PE}_{\mathrm{I}^{\prime}}\left(\sum_{i \varepsilon l^{\prime}} S S_{i}\right),
$$

onde:

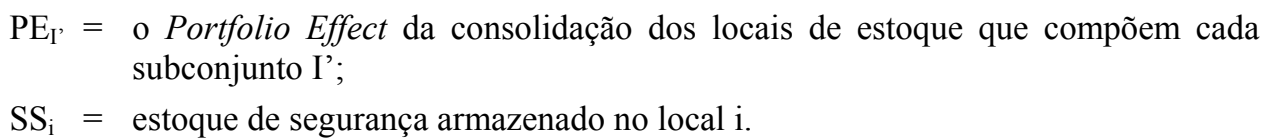

\section{b) Cálculo do Efeito da Consolidação nos Custos de Manutenção de Estoques}

Os custos de manutenção de estoques são diretamente proporcionais à quantidade e ao tempo que um produto permanece em estoque. Esse custo é composto pelos componentes relativos a custo de capital; de armazenagem e de risco. Os custos de capital podem ser avaliados pela aplicação da taxa de interesse ao capital investido em ativos em estoque e outros necessários para mantê-lo. Os custos de armazenagem incluem as taxas e os seguros dos ativos, assim como as taxas de depreciação, os custos de manutenção e reparos, condicionamento do ar, eletricidade e salários de pessoal, que podem ser contabilizados nesse item. Os custos de risco incluem exclusivamente os riscos de obsolescência, deterioração e roubos do estoque.

A Petrobras adota os seguintes valores anuais para o cálculo do custo de manutenção de estoques (Sermat, 1998):

- taxa de $15 \%$ ao ano como parcela do custo financeiro de imobilização em estoques, que é a taxa de retorno utilizada para novos investimentos;

- taxa de 3\% ao ano como medida do custo de perdas e obsolescência.

Conforme Mahmoud (1992), o ganho associado à manutenção de estoques com o custo de manutenção resultante da redução do estoque de segurança mantido e da consolidação de locais em subconjuntos I', cuja instalação centralizadora é o local $r$, é calculado pela expressão (20): 


$$
\operatorname{PHCE}_{I^{\prime}}^{r}=\left[\sum_{\mathrm{i} \in \mathrm{I}^{\prime}} \mathrm{SS}_{\mathrm{i}} \mathrm{h}_{\mathrm{i}}\right]-\left[\mathrm{h}_{\mathrm{r}}\left(1-\mathrm{PE}_{\mathrm{I}^{\prime}}\right)\left(\sum_{\mathrm{i} \in \mathrm{I}^{\prime}} \mathrm{S} S_{\mathrm{i}}\right)\right],
$$

onde:

$\mathrm{h}_{\mathrm{i}}=$ custo unitário anual de utilização do local $\mathrm{i}$;

$\mathrm{h}_{\mathrm{r}}=$ custo unitário anual de utilização do local $\mathrm{r}$ onde ocorrerá a centralização.

\section{c) Cálculo do Efeito da Consolidação nos Custos de Transportes}

Quando acontece uma centralização, o fluxo das mercadorias é alterado. O componente de custo de transporte é um fator crítico na decisão sobre centralização de estoques em alguns locais. O aumento ou decréscimo nos custos de transportes devem ser analisados considerando as distâncias e freqüências de viagens entre os fornecedores, os locais de estoque e os locais de demanda/clientes. Outros fatores a serem considerados são o volume de mercadorias movimentadas, as tarifas, as economias de escala e as condições de entrega em cada rota. No estudo de caso do E\&P-Nordeste, considerou-se que os fornecedores estão localizados na cidade de São Paulo, por ser o Estado de São Paulo onde está a grande maioria daqueles qualificados no cadastro da Petrobras. Foi considerado para fins de simplificação da análise do caso que os locais onde estão os estoques coincidem com a localização espacial da demanda. $\mathrm{O}$ efeito da centralização de estoques em $\mathrm{r}$ e de consolidação da rede de instalações nos custos de transportes $\left(\mathrm{PTCE}_{\mathrm{I}}{ }^{\mathrm{r}}\right.$ ) foi calculado medindo a variação entre esses custos nas condições descentralizada e centralizada.

Para efeito de generalização do estudo considerou-se que os fornecedores vendem seus produtos na condição FOB (Free on Board) para a Petrobras segundo a classificação INCOTERMS (Internacional Commercial Terms) de 1990. Assim, o custo de transportes, analisado por trecho fornecedor-unidade centralizadora e unidade centralizadora-clientes, será calculado, segundo Das \& Tyagi (1997), multiplicando a demanda média anual pelo custo unitário de transporte da facilidade i para o cliente j.

Os valores referentes aos custos unitários de transportes foram pesquisados junto a duas das transportadoras que oferecem serviços à Petrobras, que cederam as suas tabelas de preços para cargas fracionadas, nas quais são considerados a distância a ser percorrida, o peso e o valor do material. No estudo de caso foram utilizados os valores fornecidos pela transportadora Cinco Estrelas Ltda, que apresentou as melhores condições comerciais. Figueiredo (1999) apresenta a tabela de fretes fornecida pela transportadora e a tabela dos pesos unitários dos itens analisados. Foram extraídos os códigos utilizados pelas empresas para calcular o valor do frete em função da distância origem-destino, apresentados na Tabela I. Pode-se observar que, para os mesmos trechos, a mudança na origem-destino altera o código da tarifa, apesar das distâncias serem as mesmas, em virtude da facilidade operacional encontrada pela transportadora na rota.

Tabela I - Código de Identificação Para Cálculo do Frete em Função das Distâncias

\begin{tabular}{|c|c|c|c|}
\hline \multirow{2}{*}{ Origem } & \multicolumn{3}{|c|}{ Código (Distância em km) por Destino } \\
\cline { 2 - 4 } & Salvador & Aracaju & Mossoró \\
\hline São Paulo & $190(1901-2000 \mathrm{~km})$ & $200(2001-2200 \mathrm{~km})$ & $300(3001-3200 \mathrm{~km})$ \\
\hline Salvador & - & $35(351-400 \mathrm{~km})$ & $120(1201-1300 \mathrm{~km})$ \\
\hline Aracaju & $35(351-400 \mathrm{~km})$ & - & $95(951-1000 \mathrm{~km})$ \\
\hline Mossoró & $110(1101-1200 \mathrm{~km})$ & $75(751-800 \mathrm{~km})$ & - \\
\hline
\end{tabular}

Fonte: Transportadora Cinco Estrelas Ltda - 1998. 


\section{d) Cálculo do Efeito da Consolidação nos Custos do Processamento de Pedidos}

As premissas do modelo baseiam-se na suposição de que o tamanho do lote de compra é equivalente à demanda durante o lead time (Zin et al., 1989; 1990). Como resultado, quando estoques de um conjunto de locais distintos são consolidados, o tamanho do lote de compra que atende a essas demandas individuais será a soma das demandas durante o lead time. Isso resulta em dois tipos de economia. Primeiro, o número total de pedidos feitos pela empresa diminui para cada opção de consolidação por ciclo de ressuprimento. A redução do custo de colocar os pedidos de ressuprimento é proporcional ao fator $[\mathrm{m}-1 / \mathrm{m}]$, onde $\mathrm{m}$ é igual ao número de locais pertencentes ao conjunto consolidado (I'). Em segundo lugar, a empresa pode ressuprir um número maior de produtos a cada ciclo, o que pode resultar em economias de escala na compra, pela obtenção de descontos com uma maior quantidade comprada. $\mathrm{O}$ ganho líquido obtido pela redução no custo de compra é medido pelo Portfolio Procurement Cost Effect (PPCE $\mathrm{I}^{\mathrm{r}}$ ) que avalia exatamente a variação entre os custos antes e depois da consolidação.

No E\&P-Nordeste, o custo unitário por pedido do processo de compra nas unidades em estudo, referentes ao primeiro semestre de 1998, está mostrado na Tabela II. Esses dados se referem apenas às compras no mercado nacional, englobando $82 \%$ do total das compras da Petrobras. Em 1997, a empresa gastou com materiais e equipamentos o valor total de US\$ $1.710,00$ milhões assim distribuídos ou seja, $82 \%$ no mercado nacional (US\$ $1.405,00$ milhões) e $18 \%$ no mercado externo (US\$ 305,00 milhões).

Tabela II - Custo do Processo de Compra

\begin{tabular}{|c|c|}
\hline Unidade Operacional & Custo do Processo (R\$) \\
\hline E\&P-BA & 360,81 \\
\hline E\&P-SEAL & 270,68 \\
\hline E\&P-RNCE & 234,02 \\
\hline
\end{tabular}

Fonte: Petrobras/Sermat/Dipros - 1998

\section{e) Seleção do Esquema Ótimo de Consolidação}

Para a definição dos melhores locais das instalações centralizadoras de estoques é necessário calcular o ganho total por consolidação I', obtido pela equação:

$$
\mathrm{PCE}_{\mathrm{I}}{ }^{\mathrm{r}}=\mathrm{PHCE}_{\mathrm{I}}{ }^{\mathrm{r}}+\mathrm{PTCE}_{\mathrm{I}}{ }^{\mathrm{r}}+\mathrm{PPCE}_{\mathrm{I}}{ }^{\mathrm{r}}
$$

onde:

$\mathrm{PHCE}_{\mathrm{I}}{ }^{\mathrm{r}}{ }^{\mathrm{r}}=$ efeito pela variação do custo de manutenção de estoques;

$\mathrm{PPCE}_{\mathrm{I}}{ }^{\mathrm{r}}=$ efeito pela variação do custo de processamento de pedidos;

PTCE $_{\mathrm{I}}{ }^{\mathrm{r}}=$ efeito pela variação do ganho no custo de transportes.

A escolha do local da instalação centralizadora (r) será definida com base no valor máximo entre os $\mathrm{PCE}_{\mathrm{I}}{ }^{\mathrm{r}}$ calculados ou seja, $\mathrm{PCE}_{\mathrm{I}}{ }^{*}$, por subconjunto I'.

O esquema ótimo da consolidação é obtido pela solução de um problema de otimização combinatória similar ao problema de particionamento (set-partitioning) cuja formulação é: 
Maximizar $\sum_{k=1}^{N} P C E_{I}^{*} X_{k}$

Sujeito a $\sum_{k=1}^{N} t_{i k} X_{k}=1$, para cada $\mathrm{i}=1, \ldots, \mathrm{n}$

$X_{k}=\left\{\begin{array}{l}1, \text { se a consolidação } k \text { é selecionada no esquema ótimo; } \\ 0 \text {, de outra forma }\end{array}\right.$

onde:

$t_{i k}=$ coeficientes binários da matriz $[\mathrm{n}, \mathrm{N}]$ (vide Tabela III);

n é o número de instalações;

$\mathrm{N}$ é o número de subconjuntos de consolidações avaliadas.

No caso em estudo de $n=3$ localizações, para escolher os melhores locais para a centralização de estoques dos itens analisados a matriz inicial de seleção do esquema ótimo é aquela apresentada na Tabela III. Essa matriz é estruturada de forma que cada subconjunto de consolidações possíveis seja representado em uma única coluna. A solução ótima encontrada para o problema determina o ganho máximo a ser obtido com a consolidação das instalações e quais delas devem ser selecionadas como instalações centralizadoras por item.

Tabela III - Matriz Inicial de Seleção do Esquema Ótimo de Consolidação

\begin{tabular}{|c|ccccc|}
\hline \multicolumn{5}{|c|}{ CONSOLIDAÇÕES } \\
\hline \multirow{3}{*}{$\mathrm{PCE}_{\mathrm{I}}{ }^{*}$} & $(1,2)$ & $(1,3)$ & $(2,3)$ & $(1,2,3)$ & \\
\cline { 2 - 6 } Instalação & $\mathrm{PCE}_{12}{ }^{*}$ & $\mathrm{PCE}_{13}{ }^{*}$ & $\mathrm{PCE}_{23}{ }^{*}$ & $\mathrm{PCE}_{123}{ }^{*}$ & \\
1 & $\mathrm{X}_{1}$ & $\mathrm{X}_{2}$ & $\mathrm{X}_{3}$ & $\mathrm{X}_{4}$ & \\
2 & 1 & 1 & 0 & 1 & $=1$ \\
3 & 1 & 0 & 1 & 1 & $=1$ \\
& 0 & 1 & 1 & 1 & $=1$ \\
\hline
\end{tabular}

\section{- Solução do Problema de Consolidação de Estoques}

A solução do problema pode ser decomposta em cinco etapas:

- cálculo do percentual de redução no estoque de segurança;

- cálculo da quantidade reduzida no estoque de segurança e do efeito da consolidação nos custos de manutenção de estoques;

- cálculo do efeito da consolidação nos custos de transportes;

- cálculo do efeito da consolidação nos custos de processamento de pedidos;

- seleção do esquema ótimo de consolidação.

Foi desenvolvido um procedimento computacional para implementar essa solução em uma planilha EXCEL (Microsoft) com interface com o software de otimização What's Best (Lindo). A planilha foi organizada em cinco pastas (PE, PHCE, PTCE, PPCE, SELECTION), relativas às etapas em que a solução foi decomposta. A Figura 2 traz o esquema básico de solução do problema, identificando a entrada de dados por pasta. 


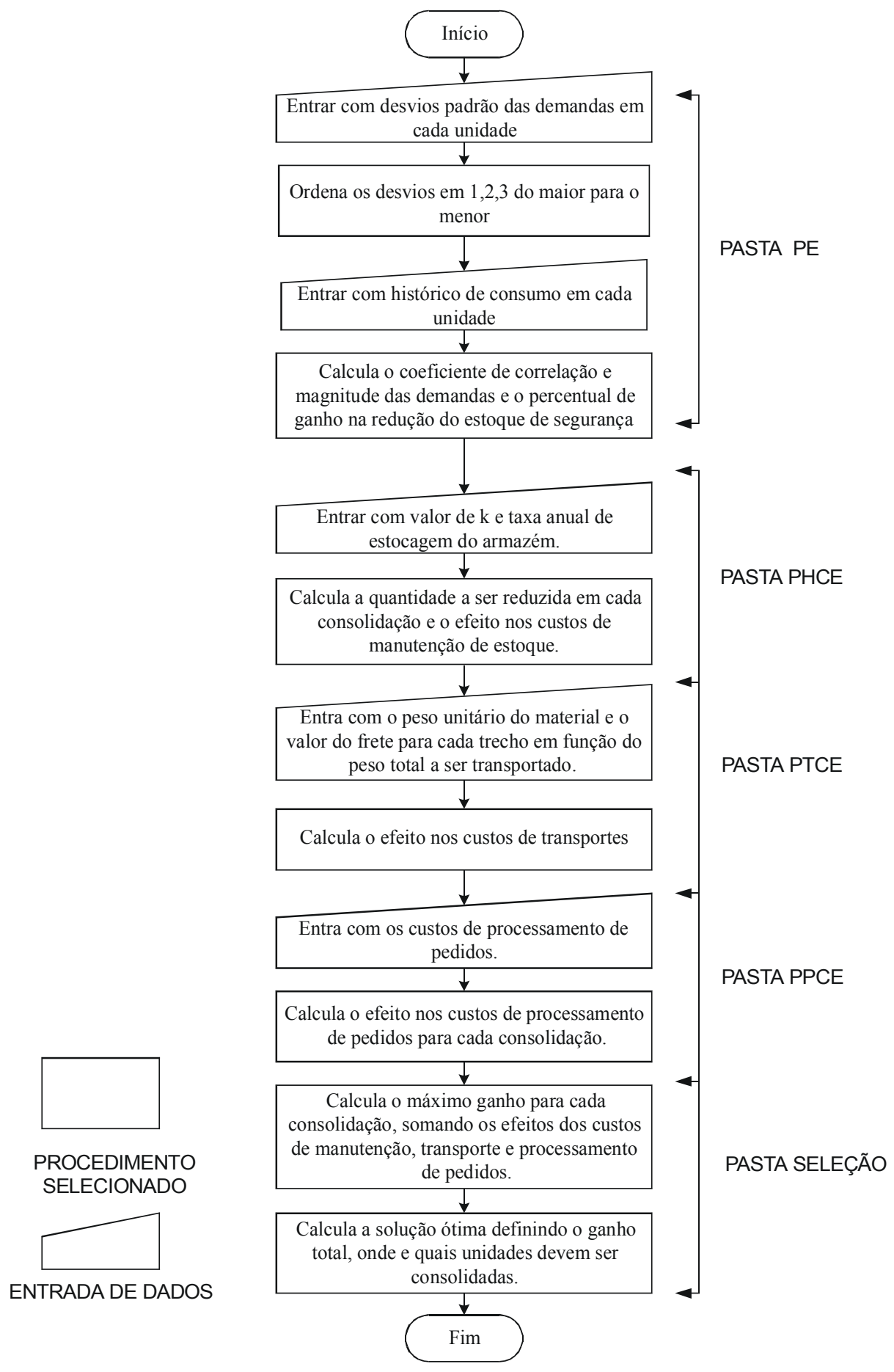

Figura II - Esquema Básico Para Solução do Problema 
Figueiredo (1999) traz detalhes sobre essas planilhas, os dados de entrada nelas introduzidos e os cálculos realizados em cada uma. Esses dados são relativos a: desvio padrão do consumo em cada unidade operacional, histórico de consumo para até 33 meses, valor do coeficiente $\mathrm{k}$ para o cálculo do estoque de segurança, preço unitário do item, peso unitário do item em $\mathrm{kg}$ e valor dos fretes para os trechos fornecedor - unidade operacional e unidade centralizadora - unidade cliente. Outros dados de entrada são relativos aos custos de processamento de pedidos por unidade operacional e a freqüência de compras por ano, além do custo de utilização dos espaços de armazenagem das unidades operacionais. Para efeito de simplificação na análise do custo logístico foi adotado para todos os itens o número de 04 (quatro) compras por ano. Para a definição do lead time foram utilizados os valores cadastrados no banco de dados de material da Petrobras. Após o preenchimento de todos os campos de entrada de dados, executados pasta a pasta na planilha, o ícone de otimização do software What's Best deve ser ativado e completa-se assim o procedimento com a geração de um relatório de saída com o ganho total obtido com a consolidação dos estoques e a seleção do melhor local para instalação da unidade centralizadora de estoques, entre os locais preexistentes.

\section{- Análise dos Resultados do Estudo de Caso}

O resultado inicial da pesquisa de levantamento de dados sobre itens comuns às três unidades identificou 80 itens cadastrados como demanda tipo Consumo (C) na classificação Petrobras, totalizando R $\$ 343.134,00$ em valores em estoque. O modelo selecionado foi aplicado aos itens classe $\mathrm{A}$, segundo a classificação $\mathrm{ABC}$, com o objetivo de avaliar a redução dos estoques de segurança e dos custos logísticos, resultantes da decisão de consolidação. A Tabela IV apresenta os resultados ótimos obtidos com a aplicação do modelo.

Tabela IV - Resultados da Centralização de Estoques

\begin{tabular}{|c|c|c|c|c|}
\hline Item & $\begin{array}{c}\text { Consolidação dos } \\
\text { E\&P'S }\end{array}$ & $\begin{array}{c}\text { Instalação } \\
\text { Centralizadora }\end{array}$ & $\begin{array}{c}\text { Ganho Anual } \\
(\mathrm{R} \$)\end{array}$ & $\begin{array}{c}\text { Redução no Estoque de } \\
\text { Segurança (\%) }\end{array}$ \\
\hline 1 & BA-SEAL-RNCE & E\&P-BA & 1704,73 & 52,4 \\
\hline 2 & BA-SEAL-RNCE & E\&P-BA & 1451,66 & 40,1 \\
\hline 3 & BA-SEAL-RNCE & E\&P-BA & 1503,53 & 43,2 \\
\hline 4 & BA-RNCE & E\&P-BA & 1920,69 & 5,3 \\
\hline 5 & BA-SEAL-RNCE & E\&P-BA & 1544,01 & 30,4 \\
\hline 6 & BA-RNCE & E\&P-BA & 1064,38 & 27,2 \\
\hline 7 & BA-SEAL & E\&P-BA & 1473,6 & 19,6 \\
\hline 8 & BA-SEAL & E\&P-BA & 1422,35 & 32,9 \\
\hline 9 & BA-SEAL & E\&P-BA & 1426,39 & 32,4 \\
\hline 10 & BA-SEAL & E\&P-BA & 1451,71 & 19,1 \\
\hline 11 & BA-SEAL & E\&P-BA & 1570,91 & 31,3 \\
\hline 12 & BA-SEAL & E\&P-BA & 1416,63 & 28,9 \\
\hline 13 & BA-SEAL & E\&P-BA & 1439,66 & 25,1 \\
\hline 14 & BA-RNCE & E\&P-RNCE & 1188,03 & 35,3 \\
\hline 15 & BA-SEAL & E\&P-BA & 1363,24 & 22,6 \\
\hline 16 & BA-SEAL & E\&P-BA & 1418,18 & 33,7 \\
\hline
\end{tabular}


Os resultados indicam potencial de redução nos custos logísticos em todos os casos analisados de consolidação de estoques. O valor total reduzido depende da política de estoques selecionada (quantidade de compras por ano e nível de serviço desejado, ou do parâmetro $\mathrm{k}$ utilizado no cálculo do estoque de segurança), freqüência de consumo e das distâncias entre unidades e entre os fornecedores e a unidade centralizadora. No caso analisado, o percentual médio conseguido com a redução nos estoques de segurança, após a consolidação, foi de $30,6 \%$, alcançando $52,6 \%$ para um determinado item. Para todos os itens analisados houve uma redução nos custos logísticos. Os custos de manutenção de estoques e de processamento de pedidos foram reduzidos em todos os casos, enquanto que na grande maioria os custos de transportes cresceram quando os locais de estocagem foram agregados.

A unidade do UN-BA foi selecionada como melhor opção para a centralização dos estoques, resultantes das consolidações, para quase a totalidade dos itens analisados. A melhor configuração, para 56\% dos itens, foi consolidar a UN-BA com UN-SEAL. Pode-se explicar esse resultado devido à menor distância da UN-BA em relação à localização dos fornecedores, estabelecida como hipótese inicial, localizados no estado de São Paulo. Além disso, pelas tabelas de fretes fornecidas pelas duas transportadoras pesquisadas, os trechos Salvador-Aracaju e Aracaju-Salvador apresentam os mesmos valores de tarifas para todas as faixas de carga, favorecendo sempre a opção de localizar o estoque mais próximo do mercado fornecedor. Além dos ganhos obtidos, a UN-BA possui melhor infra-estrutura logística do que as outras duas unidades analisadas, também possibilitando selecionar outras modalidades para o transporte de materiais de acordo com a situação de consumo (emergência ou consumo normal) e características físicas do item.

Esses resultados permitem antever reduções se a análise for estendida aos itens das demais classificações do MSM da empresa. Dessa forma, o modelo utilizado poderia ser base de metodologia para estruturar uma rede de centros de serviço ou de administração compartilhada de estoques aos quais seriam alocados determinados conjuntos de itens ou de serviços. Além disso, os valores adotados para os parâmetros do modelo e para definir a freqüência de aquisição dos materiais, que se traduzem nos resultados apresentados, utilizaram como fundamento a situação vigente na empresa para os itens Consumo (C). A variação do parâmetro $\mathrm{k}$ para o cálculo do estoque de segurança por local da rede de instalações da empresa e para a instalação centralizadora por cada item ou conjunto de itens se associa à variação no nível de serviço. A análise da variação desse parâmetro, em conjunto com a do padrão de compras por item e por unidade operacional, pode levar a uma maior ou menor agregação dos estoques por cada conjunto de itens analisados. Da mesma forma, introduzir a real distribuição espacial dos fornecedores, uma vez que se adotou uma simplificação no estudo feito, permitiria avaliar se os resultados se manteriam com origens situadas em outros estados além de São Paulo.

A Petrobras está adotando políticas que tendem à eliminação de imobilizados em estoques, ao seu posicionamento estratégico no sistema logístico e à otimização de recursos disponíveis. Nesse último caso, esses recursos seriam as unidades operacionais, sua infraestrutura de armazenagem e de gerência de estoques e o pessoal qualificado para a análise e a tomada de decisão. A combinação entre a natureza dos itens e as características do mercado deve ser observada para a formulação das políticas de suprimento da empresa. Uma vez formuladas, pode-se tentar traduzi-las em modelos analíticos que permitam visualizar aquela(s) mais adequada(s) às estratégias da corporação. A seleção e a implantação da política ótima deve considerar que ela ocasiona custos que devem ser comparados com os 
benefícios oriundos da mesma. Soluções com base em metodologias e modelos de suporte à decisão pouco sofisticados podem orientar os decisores na análise do custo e do benefício de decisões de centralização de estoques e de serviços e de otimização dos recursos disponíveis. É esse justamente o caso do modelo de centralização de Zin et al. (1989) estendido por Mahmoud (1992), que permite contrapor esses custos aos ganhos associados às economias de escala obtidas com tais decisões.

\section{Considerações Finais}

Os resultados obtidos reforçam trabalhos anteriores da Petrobras, como aquele realizado por um grupo de gerentes da empresa em novembro de 1997 na Fundação Dom Cabral, utilizando técnicas de simulação para apoiar a análise de decisão de novos projetos de investimentos. Nesse trabalho obteve-se como resultado da aplicação dessas técnicas a seleção da UN-BA como o melhor local para a instalação de uma central de administração compartilhada para a distribuição de materiais e equipamentos para o Nordeste. Além disso, o modelo aqui escolhido permite que seja analisada a possibilidade de adoção do conceito de "parcerias internas" para o gerenciamento dos estoques, apoiando a seleção da unidade operacional ótima, por região, para o gerenciamento de itens comuns.

Entretanto, o modelo selecionado considera apenas o efeito da incerteza da demanda. Para o caso da Petrobras quando as compras no mercado nacional são da ordem de $82 \%$ e os atrasos de fornecimento são penalizados contratualmente, esse modelo se mostra adequado. Entretanto, para os itens cuja aquisição acontece no mercado externo, a incerteza no tempo de ressuprimento passa a ter considerável importância. No caso da Petrobras, a maior parte do transporte internacional é feita por via marítima e o tempo médio de desembaraço aduaneiro é de 20 dias. Nesses casos o modelo de Tallon (1993), por considerar tanto as incertezas da demanda quanto do lead time, seria o mais adequado para o cálculo da redução no estoque de segurança. Entretanto, as incertezas enfrentadas pela empresa podem ser reduzidas por meio de alianças estratégicas entre clientes e fornecedores, melhorias no sistema de transportes e na comunicação entre clientes e fornecedores, pela utilização do EDI (Eletronic Data Interchange), por exemplo.

Buscou-se basicamente medir o efeito do impacto da centralização de estoques nos custos logísticos. Um aspecto que não foi medido neste trabalho são as reduções possíveis nos custos de processamento de faturas ou contas a pagar quando acontece uma centralização. Segundo pesquisa da empresa de consultoria Symnetics, a redução dos custos nos setores de contas a pagar e de contabilidade da empresa são em média $15 \%$ e $47 \%$ respectivamente, quando acontece uma centralização de atividades. Outros estudos, realizados pela Ernest \& Young, comprovaram que nessa situação o custo de processamento de contas a pagar cai de US $\$ 8,00$ para US $\$ 4,44$, conforme levantamento feito nas 100 maiores empresas assim relacionadas pela revista Fortune (Figueiredo, 1999).

Os resultados da análise realizada sugerem, para futuras pesquisas, um estudo mais abrangente considerando a possibilidade de criação de novos centros de distribuição regionais sob a filosofia da administração compartilhada de estoques, serviços e da informação nas diversas áreas de atuação da Petrobras no Brasil, tratando a empresa de forma integrada e global. Um outro aspecto importante a ser observado diz respeito a estudos sobre o comportamento da demanda para apoio ao dimensionamento dos estoques de segurança e a redução das incertezas nas estimativas de freqüências de consumo. 


\section{Agradecimentos}

Agradecemos o suporte e o financiamento que a Petrobras e o Programa CTPETRO, por meio da Agência Brasileira de Desenvolvimento Científico e Tecnológico CNPq, deram a essa pesquisa, desenvolvida no âmbito da COPPE/Universidade Federal do Rio de Janeiro.

\section{Referências Bibliográficas}

(1) Ballou, R.H. (1981). Estimating and Auditing Aggregate Inventory Levels at Multiple Stocking Points. Journal of Operations Management, 1, 143-153.

(2) Brown, R.G. (1967). Decision Rules for Inventory Management. Dryden Press, Hinsdale, IL.

(3) Chang, P.L. \& Lin, C.T. (1991). On The Effect of Centralization on Expected Costs in a Multi-Location Newsboy Problem. Journal of the Operational Research Society, 42(11), 1025-1030.

(4) Chen, M.S. \& Lin, C.T. (1989). Effects of Centralization on Expected Costs in a MultiLocation Newsboy Problem. Journal of the Operational Research Society, 40(6), 597-602.

(5) Das, C. (1978). A Reappraisal of The Square Root Law. International Journal of Physical Distribution and Materials Management, 8(2), 331-336.

(6) Das, C. \& Tyagi, R. (1997). Role of Inventory and Transportation Costs in Determining The Optimal Degree of Centralization. Transportation Research - Part E, 33(3), 171-179.

(7) Eppen, G.D. (1979). Effects of Centralization on Expected Costs in a Multi-Location Newsboy Problem. Management Science, 25(5), 498-501.

(8) Eppen, G.D. \& Martin, R.K. (1988). Determining Safety Stock in The Presence of Stochastic Lead Time and Demand. Management Science, 34(11), 1380-1390.

(9) Evers, P.T. \& Beier, F.J. (1993). The Portfolio Effect and Multiple Consolidation Points: A Critical Assessment of The Square Root Law. Journal of Business Logistics, 14(2), 109-125.

(10) Evers, P.T. (1995). Expanding The Square Root Law: An Analysis of Both Safety and Cycle Stocks. Logistics and Transportation Review, 31(1), 1-20.

(11) Figueiredo, E.D. (1999). Um Modelo de Centralização de Estoques para a Logística de Suprimento do E\&P/Petrobras. Dissertação de M.Sc., março, COPPE, Universidade Federal do Rio de Janeiro, Rio de Janeiro.

(12) Hansen, P.B. (1996). Um Método Multicriterial de Avaliação e Gestão de Processos Produtivos da Indústria de Propriedade Contínua. Dissertação de M.Sc., Engenharia de Produção, Universidade Federal do Rio Grande do Sul, Porto Alegre.

(13) Heskett, J.L.; Glaskowsky, Jr., N.A. \& Ivie, R.M. (1973). Business Logistics. The Ronald Press Company, New York. 
(14) Lambert, D.M. \& Stock, J.R. (1992). Strategic Logistics Mangement. Richard D. Irwin, Inc., $3^{\mathrm{a}}$ ed.

(15) Mahmoud, M.M. (1992). Optimal Inventory Consolidation Schemes: A Portfolio Effect Analysis. Journal of Business Logistics, 13(1), 193-214.

(16) Maister, D.H. (1976). Centralization of Inventories and The Square Root Law. International Journal of Physical Distribution and Materials Management, 6(3), 124-134.

(17) Meredith, J.R. (1992). The Management of Operations: A Conceptual Empahasis. John Wiley \& Sons, Inc., New York, $4^{\mathrm{a}}$ ed.

(18) Mirchandani, P.B. \& Francis, R.L. (1990). Discrete Location Theory, Wiley Interscience, New York.

(19) Mood, A.M.; Graybill, F.A. \& Boes, D.C. (1974). Introduction to the Theory of Statistics. Mcgraw-Hill Book Co., New York, 178-179.

(20) PPB (1997). Purchasing Performance Benchmarks for the U.S. Petroleum Industry. Center for Advanced Purchasing Studies - CAPS.

(21) Sermat (1997). Custos de Suprimento de Materiais e Equipamentos. Serviço de Material, Petrobras.

(22) Sermat (1998). Custos de Suprimento de Materiais e Equipamentos. Serviço de Material, Petrobras.

(23) Silver, E.A. \& Peterson, R. (1985). Decision Systems for Inventory Management and Production Planning. Jonh Wiley \& Sons, New York.

(24) Smykay, E.W. (1973). Physical Distribution Management. Macmillan Publishing Co., New York.

(25) Starr, M.K. \& Miller, D.W. (1962). Inventory Control: Theory and Practice. PrenticeHall, Englewood Cliffs, NJ.

(26) Tallon, W.J. (1993). The Impact of Inventory Centralization on Aggregate Safety Stock: The Variable Supply Lead Time Case. Journal of Business Logistics, 14(1), 185-203.

(27) Zin, W.; Levy, M. \& Bowersox, D.J. (1989). Measuring The Effect of Inventory Centralization/Decentralization on Aggregate Safety Stock: The "Square Root Law" Revisited. Journal of Business Logistics, 10(1), 1-14.

(28) Zin, W. \& Marmorstein, H. (1990). Comparing Two Alternative Methods of Determining Safety Stock Levels: The Demand and The Forecast Systems. Journal of Business Logistics, 11(1), 95-110.

(29) Zin, W.; Levy, M. \& Bowersox, D.J. (1990). On Assumed Assumptions and The Inventory Centralization/Decentralization Issue. Journal of Business Logistics, 11(2), 139-142. 